\title{
Image current heating on a metal surface due to charged bunches
}

\author{
Xintian E. Lin and David H. Whittum \\ Stanford Linear Accelerator Center, Stanford University, Stanford, California 94309
}

(Received 18 February 2000; published 5 October 2000)

\begin{abstract}
When charged particles pass through a metal pipe, they are accompanied by an image current on the metal surface. With intense short bunches passing near or even into the metal surface, the peak image current density can be very high. This current may result in substantial temperature rise on the surface, especially in high peak current, multibunch operation. In this paper, we derive an explicit formula for the surface temperature rise due to this previously unrecognized pulsed heating effect and show that this effect dominates the proposed linear coherent light source collimator spoiler and wire scanner heating. Without proper account, it can result in component and instrument failures. The result also applies to optical transition radiation screens, profile screens, wire scanners, exit windows, and targets, which the beam crosses.
\end{abstract}

PACS numbers: 41.20.-q, 44.10.+i, 29.27.-a, 41.85.Si

\section{INTRODUCTION}

In high energy accelerators, beams are brought to the collision point and collision products are detected. To reduce background noise, well-defined beams are highly desirable. However, due to intrabeam and interbeam interaction, the beams at the end of the long accelerator usually have distorted shapes and beam halo may form as well. Therefore, a collimation section is placed before the interaction point, as illustrated in Fig. 1. Damage to the collimator surface, which is observed in Stanford linear collider operation [1], is a primary consideration in engineering design. For effective collimation, the aperture $2 b$ is usually chosen to be about $10 \sigma_{x}$. The choice of beam transverse size $\sigma_{x}$ at collimation, and consequently $2 b$, is then restricted by several factors, including wakefields generated by the small aperture, possible damage to the collimator due to the energy density of the beams, and magnet designs to achieve the proper beta function. The damage to the collimator and any other materials the beams run into are usually accounted for by the radiation from charged particles passing through solid material [2]. In this article we introduce another effect that limits the peak current and beam size. It is a result of the pulsed surface heating induced on the collimator surface by the image current. We also show in the Appendix that the result extends to other beam metal surface contact in accelerator systems, such as optical transition radiation (OTR) screens, profile screens, wire scanners, beam exit windows, targets, etc.
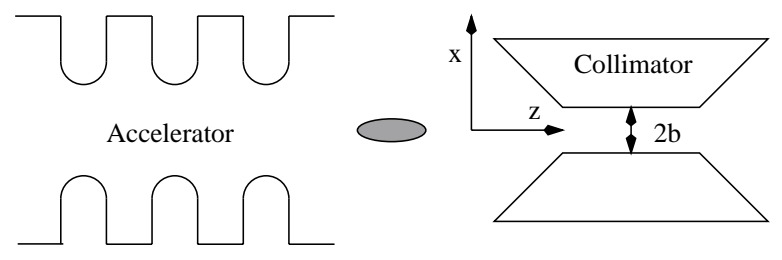

FIG. 1. Post-linac collimation.

\section{SINGLE BUNCH PULSED HEATING}

Taking a cylindrical pipe, for example (Fig. 2), the maximum surface magnetic field due to a relativistic charged bunch moving in the $z$ direction is

$$
H_{x}(x=0, y=0)=-\frac{I}{2 \pi}\left(\frac{1}{a}+\frac{1}{r_{0}} \frac{r_{0}-a}{a}\right),
$$

where $a$ is the distance from the pipe wall, and the bunch current $I$ can be written in the form

$$
I(z, t)=\frac{Q c}{\sqrt{2 \pi} \sigma_{z}} e^{-(z-c t)^{2} / 2 \sigma_{z}^{2}},
$$

where $Q$ represents bunch charge. A Gaussian bunch with length $\sigma_{z}$ is assumed. In the limit of $a \ll r_{0}$, the field is well approximated by that of an infinite flat surface (Fig. 3),

$$
H_{x}(x, y=0)=-\frac{I}{\pi} \frac{a}{a^{2}+x^{2}} .
$$

Inside metals, the displacement current term $\partial D / \partial t$ is negligible, Maxwell's equations reduce to a diffusion equation

$$
\nabla^{2} H_{x}=-\sigma \mu_{0} \frac{\partial H_{x}}{\partial t}
$$

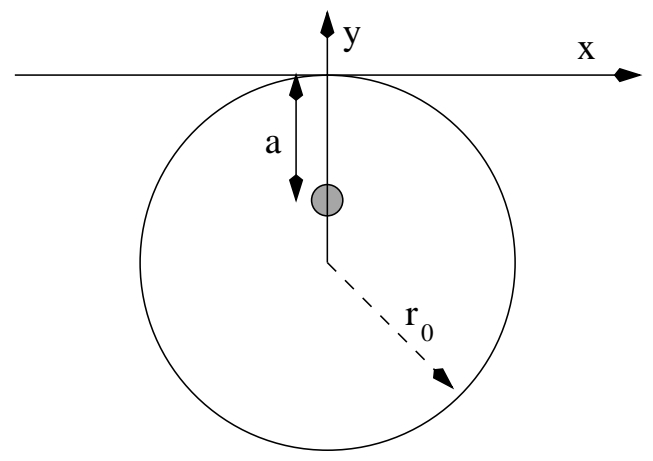

FIG. 2. Electron bunch passing through a smooth pipe.

(C) 2000 The American Physical Society

$101001-1$ 


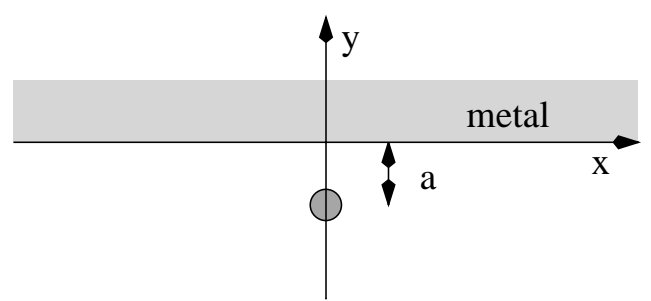

FIG. 3. Electron bunch passing close to a surface pipe.

The electric conductivity $\sigma$ is $5.8 \times 10^{7} / \Omega \cdot \mathrm{m}$ for copper. In the frequency domain, we have

$$
\left(\frac{\partial^{2}}{\partial x^{2}}+\frac{\partial^{2}}{\partial y^{2}}+\frac{\partial^{2}}{\partial z^{2}}\right) \tilde{H}_{x}=j \omega \sigma \mu \tilde{H}_{x}
$$

with boundary condition from Eq. (3),

$$
\tilde{H}_{x}(x, y=0, z, \omega)=-\frac{\tilde{I}(z, \omega)}{\pi} \frac{a}{a^{2}+x^{2}},
$$

where the Fourier transform of current

$$
\tilde{I}(z, \omega)=\int_{-\infty}^{+\infty} I(z, t) e^{-j \omega t} d t=Q e^{-\omega^{2} / 2 \omega_{\sigma}^{2}} e^{-j \omega z / c} .
$$

The characteristic frequency spread $\omega_{\sigma}=c / \sigma_{z}$. An order of magnitude estimation gives $\partial^{2} / \partial x^{2} \approx 1 / a^{2}$ and $\partial^{2} / \partial z^{2} \approx 1 / \sigma_{z}^{2}$. With $a \approx 1 \mathrm{~mm}, \sigma_{z} \approx 125 \mu \mathrm{m}$, and $\omega_{\sigma} \sigma \mu \approx 1 /(80 \mathrm{~nm})^{2} \gg\left(1 / a^{2}, 1 / \sigma_{z}^{2}\right)$, Eq. (5) simplifies to

$$
\frac{\partial^{2}}{\partial y^{2}} \tilde{H}_{x}=j \omega \sigma \mu \tilde{H}_{x} .
$$

Together with Eq. (6), we obtain the solution

$$
\tilde{H}_{x}(x, y>0, z, \omega)=-\frac{\tilde{I}(z, \omega)}{\pi} \frac{a}{a^{2}+x^{2}} e^{-[(1+j) / \delta] y},
$$

with skin depth $\delta=\sqrt{2 / \omega \mu \sigma}$.

The current density $\tilde{J}_{z}$ inside the metal is related to the magnetic field by

$$
\tilde{J}_{z}=\frac{\partial \tilde{H}_{x}}{\partial y}=\tilde{H}_{x}\left(-\frac{1+j}{\delta}\right) .
$$

The resulting Ohmic heating, $\left|\tilde{J}_{z}\right|^{2} / \sigma$, gives rise to a power spectrum density

$$
\tilde{P}_{d}(x, y, z, \omega)=\left(\frac{|\tilde{I}(z, \omega)|}{\pi}\right)^{2}\left(\frac{a}{a^{2}+x^{2}}\right)^{2} \frac{1}{\sigma} \frac{2}{\delta^{2}} e^{-2 y / \delta},
$$

which is integrated over frequency to obtain the total energy density deposited by a single bunch,

$$
\begin{aligned}
E_{d}(x, y, z) & =\int_{-\infty}^{+\infty} \tilde{P}_{d}(x, y, z, \omega) \frac{d \omega}{2 \pi} \\
& =\frac{Z_{0} c}{2 \pi}\left(\frac{Q}{\sigma_{z}}\right)^{2} \frac{1}{\pi^{2}}\left(\frac{a}{a^{2}+x^{2}}\right)^{2} g\left(y / \delta\left(\omega_{\sigma}\right)\right) .
\end{aligned}
$$

The penetration function

$$
\begin{aligned}
g(u) & =\int_{0}^{\infty} e^{-z} e^{-2 u z^{1 / 4}} d z \\
& =\frac{2}{(2 \pi)^{3 / 2}} G_{4,1}^{1,4}\left[\begin{array}{l|l}
\frac{u^{4}}{16} & {[0]} \\
{[0,1 / 4,1 / 2,3 / 4]}
\end{array}\right]
\end{aligned}
$$

is expressed in terms of Meijer's $G$ function [3]. The values of $g(u)$ and $e^{-2 u}$ are plotted in Fig. 4. There are a few points worth noting. First, $g(0)=1$. Second, at large $u, g(u) \rightarrow 3 / 2 u^{4}$. The penetration function has a longer tail than the exponential. Comparing the integrals $\int_{0}^{\infty} g(u) d u=\Gamma(3 / 4) / 2 \approx 0.6127$ and $\int_{0}^{\infty} e^{-2 u} d u=0.5$, it is clear that $e^{-2 u}$ underestimates the total energy by about $18 \%$. For a better approximation of $g(u)$, we may use $e^{-2 u / \Gamma(3 / 4)}$, i.e., replacing the argument $y / \delta\left(\omega_{\sigma}\right)$ by $y / \delta_{e}$, where the effective skin depth $\delta_{e}=\Gamma(3 / 4) \delta\left(\omega_{\sigma}\right)$ accounts for the greater penetration. The exact and approximate solutions are compared in Fig. 5. We will use the effective skin depth in later sections.

In writing Eq. (12), we have ignored the heat diffusion over the time scale $\Delta t \approx \sigma_{z} / c$. This is a good approximation as long as thermal diffusion length is much smaller than the rf skin depth; i.e.,

$$
l_{\mathrm{diff}}=\sqrt{\frac{K}{\rho C} \Delta t}=\sqrt{\frac{K}{\rho C} \frac{\sigma_{z}}{c}} \ll \delta\left(\omega_{\sigma}\right)=\sqrt{\frac{2}{\mu \sigma} \frac{\sigma_{z}}{c}},
$$

which reduces to

$$
\sqrt{\frac{K \mu \sigma}{2 \rho C}} \ll 1
$$

where $K, \rho$, and $C$ are the thermal conductivity, density, and specific heat $(\mathrm{J} / \mathrm{g})$, respectively. Taking copper and titanium for example, $\sqrt{K \mu \sigma / 2 \rho C}=0.065$ and $3.8 \times$ $10^{-3}$, respectively. This shows that Eq. (14) is an excellent assumption for most metals since copper has one of the highest $K$ and $\sigma$ of all metals.

To a very good approximation, the single bunch pulsed heating can be considered as an instantaneous energy deposition. The instantaneous temperature rise is then

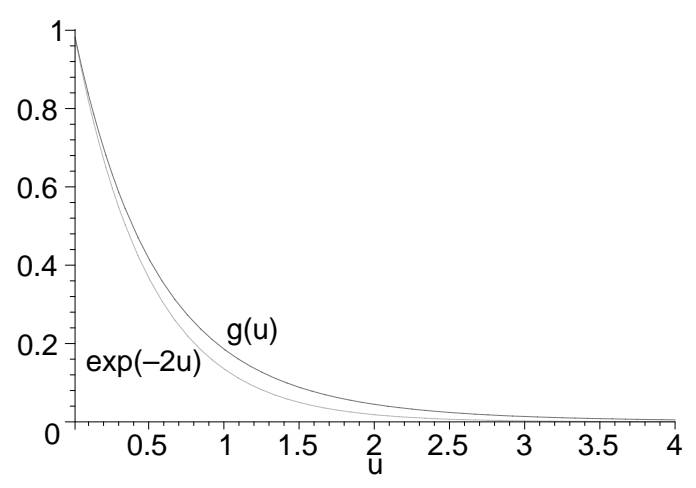

FIG. 4. Penetration function $g(u)$ and $e^{-2 u}$. 


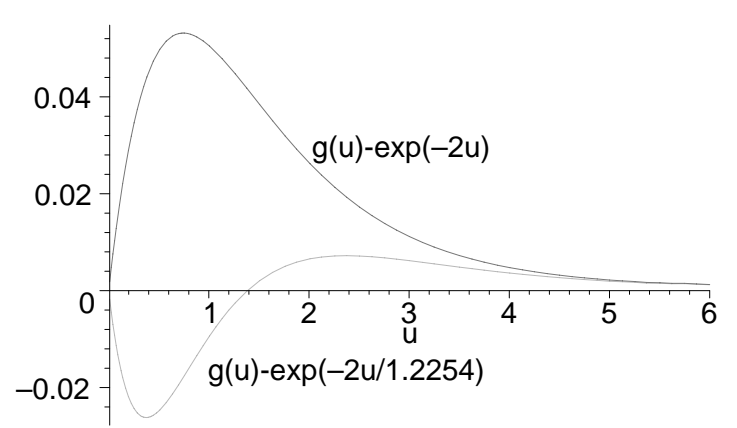

FIG. 5. Difference between $g(u)$ and $e^{-2 u}, e^{-2 u / 1.2254}$.

$$
\begin{aligned}
T_{1}(x, y, z) & =\frac{E_{d}(x, y, z)}{\rho C} \\
& =\frac{Z_{0} c}{2 \pi}\left(\frac{Q}{\sigma_{z}}\right)^{2} \frac{1}{\pi^{2}}\left(\frac{a}{a^{2}+x^{2}}\right)^{2} \frac{g\left(y / \delta\left(\omega_{\sigma}\right)\right)}{\rho C} .
\end{aligned}
$$

It is notable that the peak temperature is independent of the electrical conductivity. The resistive loss and penetration depth are both inversely proportional to the square root of the conductivity; therefore, the energy density is a constant. In one future collider design [4], a titanium spoiler with $r_{0}=2 \mathrm{~mm}$ is located in the post-linac collimation section. Taking $Q=1.52 \mathrm{nC}, \sigma_{z}=125 \mu \mathrm{m}$, $\rho C=2.36 \mathrm{~J} / \mathrm{cm}^{3}$ [5] and assuming $a=r_{0}$, Eq. (16) gives $T_{1}(0)=0.029^{\circ} \mathrm{C}$. In fact, when the beam is at the center of the circular pipe, Eq. (3) overestimates surface magnetic field by a factor of 2 compared with Eq. (1). The temperature rise should be $T_{1}(0)=0.007^{\circ} \mathrm{C}$.

\section{MAXIMUM SINGLE BUNCH PULSE HEATING}

The above example shows that, under normal conditions, such a spoiler is quite safe. However, the spoiler is also part of the machine protection system and is required to survive, in the worst case, a full bunch train impact. Such an event corresponds to beam missteering/dumping or equipment failure [4]. Under those circumstances, the electron bunches can get very close to, or even into, the metal surface.

Let us consider a bunch with Gaussian transverse distribution (Fig. 6)

$$
\tilde{I}(x, y, z, \omega)=\tilde{I}(z, \omega) \frac{e^{-x^{2} / 2 \sigma_{x}^{2}}}{\sqrt{2 \pi} \sigma_{x}} \frac{e^{-(y+a)^{2} / 2 \sigma_{y}^{2}}}{\sqrt{2 \pi} \sigma_{y}} .
$$

Since the electron bunch current in the region $y>0$ is neutralized by a corresponding image current inside the metal, they do not contribute to the magnetic field at $y=0$. And, due to its low current density compared to the surface image current density $\left(1 / \sigma_{y}\right.$ versus $\left.1 / \delta\right)$, the heating effect is also negligible. Radiation heating, primarily ionization at short distance [6], from electrons passing through metal is an important source, which is considered at the end of this paper. From Eq. (3), the surface magnetic field reads

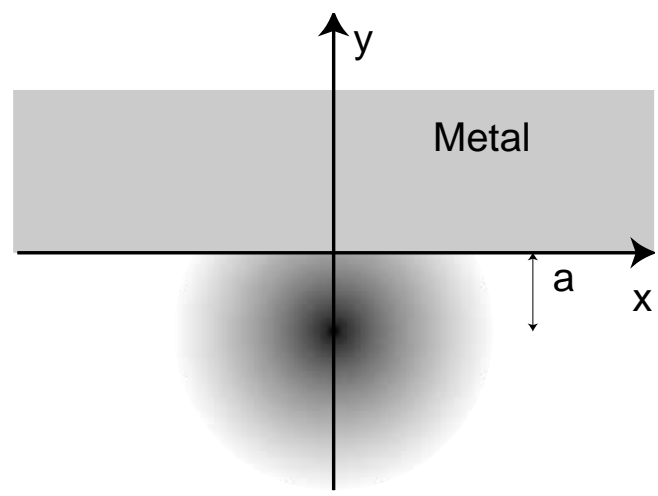

FIG. 6. Electron beam running into a metal surface.

$$
\tilde{H}_{x}(x=0, y=0, z, \omega)=\int_{-\infty}^{0} d y \int_{-\infty}^{+\infty} \frac{\tilde{I}}{\pi} \frac{y d x}{y^{2}+x^{2}} .
$$

Integrating in $x$, we have

$$
\tilde{H}_{x}(x=0, y=a, z, \omega)=-\frac{\tilde{I}(z, \omega)}{\pi \sqrt{\sigma_{x} \sigma_{y}}} f\left(\frac{a}{\sigma_{x}}, \frac{\sigma_{y}}{\sigma_{x}}\right),
$$

where

$$
\begin{aligned}
f(u, v) & =\frac{1}{2 \sqrt{v}} \int_{0}^{\infty} e^{-\left[(u-w)^{2} / 2 v^{2}\right]} e^{w^{2} / 2} \operatorname{erfc}\left(\frac{w}{\sqrt{2}}\right) d w \\
\operatorname{erfc}(w) & =\frac{2}{\sqrt{\pi}} \int_{w}^{\infty} e^{-t^{2}} d t
\end{aligned}
$$

In particular,

$$
f(u, 1)=\frac{1}{2 u}\left[\operatorname{erfc}\left(-\frac{u}{\sqrt{2}}\right)-e^{-u^{2} / 2}\right] .
$$

The value of $f(u, v)$ has one maximum as a function of $u$ for every $v$. The maxima may be found numerically, and its value $f_{\max }$ is fitted to the following function:

$$
f_{\max }(v) \approx \alpha \sqrt{\frac{1}{2 \pi}} \frac{\log (1+\pi v)}{\sqrt{v}} .
$$

The parameter $\alpha$ is nominally 1 , which gives the exact asymptotic limit for small and large $v$. Over the entire range of $0<v<\infty$, this approximation gives the upper limit of $f_{\max }$ and is never more than $5.6 \%$ off. To be more accurate, a value of $\alpha=0.95$ produces no more than $0.5 \%$ relative error over the range of $[0.25,250]$. A numerical solution of $\alpha$ is plotted in Fig. 7 for reference, and, in particular, $f_{\max }(1)=0.54$ for a round beam.

Following Eq. (19), the maximum magnitude of the surface magnetic field becomes

$$
\tilde{H}_{x}^{\max }=\frac{\tilde{I}(z, \omega)}{\pi} \frac{f_{\max }\left(\frac{\sigma_{y}}{\sigma_{x}}\right)}{\sqrt{\sigma_{x} \sigma_{y}}} .
$$




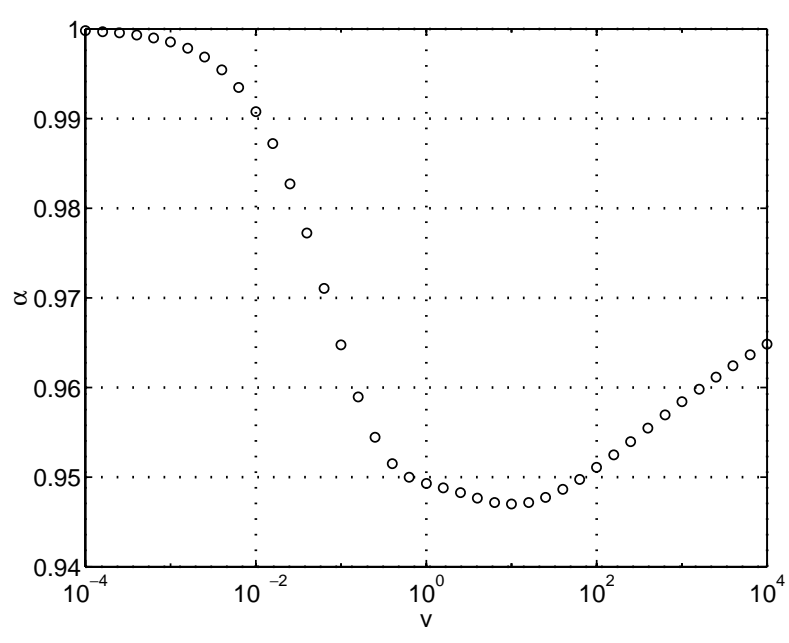

FIG. 7. Plot of the exact value of $\alpha$ as a function of $v$.

The corresponding maximum surface temperature rise due to single bunch heating is

$$
T_{1}^{\max }=\frac{Z_{0} c}{2 \pi}\left(\frac{Q}{\sigma_{z}}\right)^{2} \frac{f_{\max }^{2}\left(\frac{\sigma_{y}}{\sigma_{x}}\right)}{\pi^{2} \sigma_{x} \sigma_{y}} \frac{1}{\rho C} .
$$

At the titanium spoiler, the transverse beam size $\sigma_{y}=$ $60 \mu \mathrm{m}$ and $\sigma_{x}=160 \mu \mathrm{m}$, giving a pulsed temperature rise of $T_{1}^{\max }=2.83^{\circ} \mathrm{C}$.

\section{MULTIBUNCH HEATING}

Most of the new generation of accelerators are running in multibunch mode for efficiency and luminosity reasons. A nominal configuration calls for ninety $125 \mu \mathrm{m}$ long bunches spaced by about $1.4 \mathrm{~ns}$. This bunch train, i.e., pulse, is repeated 120 times per second, as illustrated in Fig. 8.

In Sec. II we showed that for a single bunch the thermal diffusion length $l_{\text {diff }}$ is always smaller than the rf penetration depth $\delta_{e}$. Therefore, thermal diffusion is negligible.

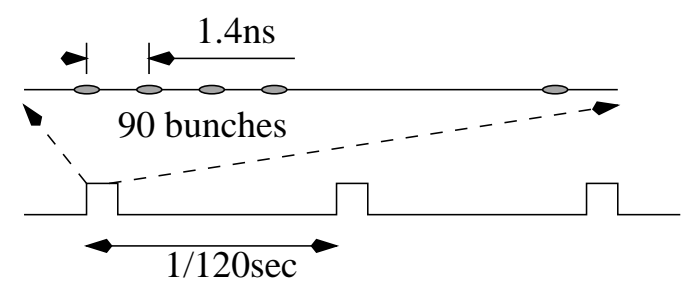

FIG. 8. Bunch structure in the modern collider.

In the multiple bunch scheme, there are three regions of operation.

\section{A. Short thermal diffusion length}

If the thermal diffusion length of the bunch train is smaller than the rf penetration depth $\delta_{e}$, i.e.,

$$
l_{\mathrm{diff}}^{\mathrm{train}}=\sqrt{\frac{K N_{p} t_{p}}{\rho C}} \ll \delta_{e}=\Gamma(3 / 4) \sqrt{2 \sigma_{z} / c \mu \sigma},
$$

then

$$
T_{N}(y)=N_{p} T_{1}(y)
$$

where $N_{p}$ is the number of bunches in a train and $t_{p}$ is the bunch separation. Here we have considered only the bunches within one pulse. The $120 \mathrm{~Hz}$ pulse structure is of secondary concern because the heat has mostly dissipated before the next bunch train arrives.

\section{B. Long thermal diffusion length}

However, in the above configuration, $l_{\text {diff }}^{\text {train }}$ is $3.8 \mu \mathrm{m}$ and $1.1 \mu \mathrm{m}$ for copper and titanium, respectively, while the effective skin depth $\delta_{e}$ is $0.117 \mu \mathrm{m}$ and $0.558 \mu \mathrm{m}$, respectively. To the first order, we may assume $l_{\text {diff }}^{\text {train }} \gg$ $\delta_{e}$ and treat all the energy as deposited on the surface $y=0$. This overestimates the surface temperature slightly.

From Eq. (11), we can obtain the energy flux $E_{f}$ deposited on the metal surface by a single bunch

$$
\begin{aligned}
E_{f}(x, y=0, z) & =\int_{-\infty}^{+\infty} \frac{d \omega}{2 \pi} \int_{0}^{\infty} \tilde{P}_{d} d y=\left(\frac{Q}{\pi}\right)^{2}\left(\frac{a}{a^{2}+x^{2}}\right)^{2} \int_{-\infty}^{+\infty} \sqrt{\frac{\omega \mu}{2 \sigma}} e^{-\omega^{2} / \omega_{\sigma}^{2}} \frac{d \omega}{2 \pi} \\
& =\left(\frac{Q}{\pi}\right)^{2}\left(\frac{a}{a^{2}+x^{2}}\right)^{2} \sqrt{\frac{\mu}{2 \sigma}}\left(\frac{c}{\sigma_{z}}\right)^{3 / 2} \frac{\Gamma(3 / 4)}{2 \pi}
\end{aligned}
$$

The one-dimensional heat diffusion solution of a point source $E_{f} \delta(y) \delta(t)$ is

$$
T(y, t)=\frac{E_{f}}{\sqrt{\pi K \rho C t}} e^{-y^{2} / 4 D t},
$$

where diffusion constant $D=K / \rho C$. We can write the multibunch result as

$$
T_{N}(y, t)=\sum_{n=1}^{N_{p}} E_{f} \frac{e^{-y^{2} / 4 D\left(t-n t_{p}\right)}}{\sqrt{\pi K \rho C\left(t-n t_{p}\right)}} H\left(t-n t_{p}\right),
$$

where $H(t)$ is a step function with $H=1$ for $t>0$, and zero otherwise. When $N_{p}$ is large, the sum is approximated by an integral

$$
T_{N}(y, t)=\int_{0}^{t} \frac{E_{f} e^{-y^{2} / 4 D\left(t-t^{\prime}\right)}}{\sqrt{\pi K \rho C\left(t-t^{\prime}\right)}} H\left(N_{p} t_{p}-t^{\prime}\right) \frac{d t^{\prime}}{t_{p}},
$$

which yields the surface temperature rise

$$
T_{N}\left(0, N_{p} t_{p}\right)=\frac{2 \sqrt{N_{p}} E_{f}}{\sqrt{\pi K \rho C t_{p}}} .
$$


Using Eq. (27) and substituting $a /\left(a^{2}+x^{2}\right)$ by $f_{\max }\left(\sigma_{y} / \sigma_{x}\right) / \sqrt{\sigma_{x} \sigma_{y}}$, we have the maximum temperature rise induced by a multibunch train,

$$
T_{N}^{\max }=\frac{2 \sqrt{N_{p}} Q^{2}}{\sqrt{\pi K \rho C t_{p}}} \frac{f_{\max }^{2}\left(\frac{\sigma_{y}}{\sigma_{x}}\right)}{\pi^{2} \sigma_{x} \sigma_{y}} \sqrt{\frac{\mu}{2 \sigma}}\left(\frac{c}{\sigma_{z}}\right)^{3 / 2} \frac{\Gamma\left(\frac{3}{4}\right)}{2 \pi} .
$$

The result has a characteristic $\sqrt{N_{p}}$ growth of a diffusion solution. Taking the titanium collimator design as an example, the maximum multibunch heating will be $T_{N}^{\max }=83^{\circ} \mathrm{C}$.

\section{Thermal diffusion length comparable with rf penetration depth}

In the previous section we assume that the diffusion length $l_{\text {diff }}^{\text {train }}$ is much larger than the rf penetration depth $\delta_{e}$, therefore the energy is treated as if deposited on the metal surface at $y=0$. A more accurate calculation takes the rf penetration into account. The temperature rise at $y=0$ due to a delta pulse energy density $\delta\left(y-y^{\prime}\right) \delta\left(t-t^{\prime}\right)$ is [7]

$$
T\left(0, t, y^{\prime}, t^{\prime}\right)=\frac{1}{\sqrt{\pi K \rho C\left(t-t^{\prime}\right)}} e^{-y^{\prime 2} / 4 D\left(t-t^{\prime}\right)} .
$$

Then the surface temperature rise due to a single bunch is

$$
T_{1}\left(0, t, t^{\prime}\right)=\int_{0}^{\infty} E_{d}\left(y^{\prime}\right) \frac{e^{-y^{\prime 2} / 4 D\left(t-t^{\prime}\right)}}{\sqrt{\pi K \rho C\left(t-t^{\prime}\right)}} d y^{\prime} .
$$

From Eq. (12) we have $E_{d}(y)=E_{d}(0) g\left(y / \delta_{\sigma}\right)$. Using the exponential approximation for $g$, we can integrate Eq. (34) to obtain

$$
\begin{aligned}
T_{1}\left(0, t, t^{\prime}\right) & =\frac{E_{d}(0)}{\rho C} e^{\hat{x}^{2}} \operatorname{erfc}(\hat{x}), \\
\hat{x} & =\sqrt{4 D\left(t-t^{\prime}\right)} / \delta_{e} .
\end{aligned}
$$

At $t=t^{\prime}$, it reduces to Eq. (16). Using the expansions

$$
e^{x^{2}} \operatorname{erfc}(x)=\frac{1}{\sqrt{\pi} x}\left[1+\sum_{m=1}^{\infty} \frac{(2 m-1) ! !}{\left(-2 x^{2}\right)^{m}}\right],
$$

Eq. (35) becomes

$$
T_{1}\left(0, t, t^{\prime}\right)=\frac{E_{d}(0) \delta_{e} / 2}{\sqrt{\pi D\left(t-t^{\prime}\right)} \rho C}\left[1+\sum_{m=1}^{\infty} \frac{(2 m-1) ! !}{\left(-2 \hat{x}^{2}\right)^{m}}\right] .
$$

Noting that $E_{d}(0) \delta_{e} / 2$ equals the total energy flux deposited by the bunch, the first term of Eq. (37) reproduces the point source result. In this particular case, the diffusion length in Ti between pulses, $\sqrt{D t_{p}}=0.114 \mu \mathrm{m}$, is smaller than the effective skin depth $\delta_{e}=0.558 \mu \mathrm{m}$; the expansion does not converge very well for small $\hat{x}$. So we take Eq. (35) and sum over all the bunches,

$$
\begin{aligned}
T_{N}(0, t) & =\frac{E_{d}(0)}{\rho C} \sum_{n=0}^{N_{p}-1} e^{x_{n}^{2}} \operatorname{erfc}\left(\hat{x}_{n}\right) H\left(t-n t_{p}\right), \\
\hat{x}_{n} & =\sqrt{4 D\left(t-n t_{p}\right)} / \delta_{e} .
\end{aligned}
$$

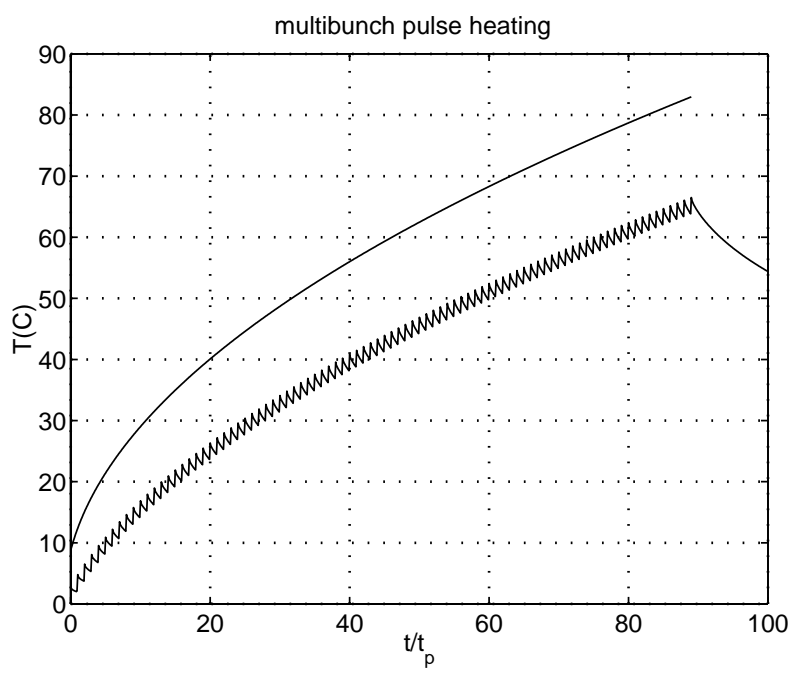

FIG. 9. Multibunch electron bunch heating for $N_{p}=90$. The smooth approximation is from Eq. (32).

The result, plotted in Fig. 9, gives $T_{N}^{\max }=66.5^{\circ} \mathrm{C}$. It is about $20 \%$ lower than the simple estimation from Eq. (32).

\section{SUMMARY}

We have derived the image current heating on the metal surface due to charged pulses. The results are summarized in Table I.

The pulsed heating due to charged bunches can be substantial with high peak current, small beam/structure size, and multiple bunches. A future linear collider spoiler design is shown to give $66.5^{\circ} \mathrm{C}$ temperature rise when a bunch train approaches it very closely. This result should be compared to the corresponding radiation heating from electromagnetic showering and ionization. Assuming the spoiler is shorter than a radiation length and there is no thermal diffusion, i.e., $\sigma_{x, y} \gg l_{\text {diff }}^{\text {train }}$, the peak temperature is

$$
T_{\mathrm{rad}}=\frac{N_{p}(Q / e) d E / d x}{2 \pi \sigma_{x} \sigma_{y} \rho C} .
$$

TABLE I. Electron pulse heating from image current

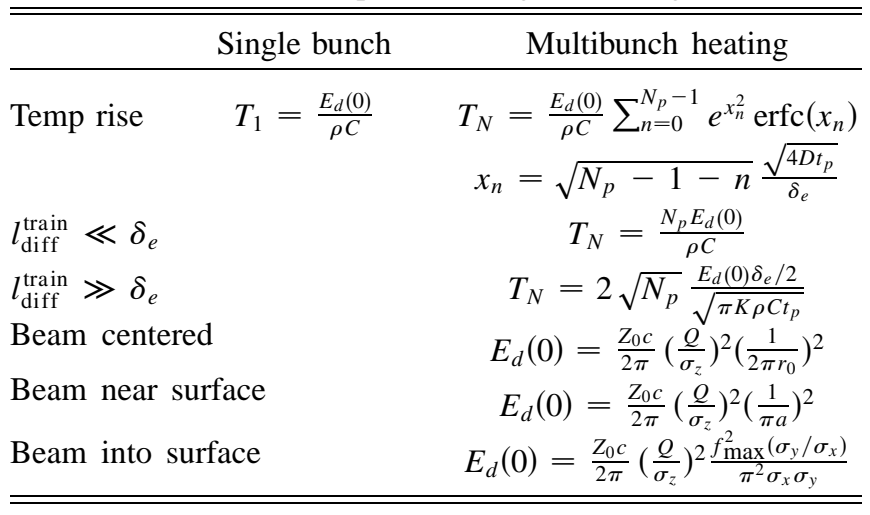


The energy deposition per unit length is $d E / d x=$ $7.2 \mathrm{MeV} / \mathrm{cm}$ [2] for titanium and the resulting $T_{\mathrm{rad}}=$ $728^{\circ} \mathrm{C}$. Under these parameters, radiation heating is much higher than that from image currents. But it is notable that electron pulse heating $T_{N}^{\max }$ is proportional to $\left(Q / \sigma_{z}\right)^{2}$ because of $I^{2} R$ heating, while radiation heating is linear in $Q$. For the case of high peak current, image current heating will dominate. Taking the linear coherent light source (LCLS) as an example, the beam parameters are $Q=$ $1 \mathrm{nC}, \sigma_{x}=\sigma_{y}=38 \mu \mathrm{m}$, and $\sigma_{z}=20 \mu \mathrm{m}$ [8]. The Ti spoiler will suffer a pulsed temperature rise of $T_{1}^{\max }=$ $390{ }^{\circ} \mathrm{C}$ and $T_{\text {rad }}=34{ }^{\circ} \mathrm{C}$ when a bunch strikes it. Similar calculations on the stainless steel undulator vacuum chamber yield $T_{1}^{\max }=324{ }^{\circ} \mathrm{C}$ and $T_{\text {rad }}=45^{\circ} \mathrm{C}(Q=1 \mathrm{nC}$, $\sigma_{x}=\sigma_{y}=32 \mu \mathrm{m}, \quad \sigma_{z}=20 \mu \mathrm{m}, \quad \rho C=4.0 \mathrm{~J} / \mathrm{cm}^{3}$, and $d E / d x=11.7 \mathrm{MeV} / \mathrm{cm})$. The consequence of these temperature rises is not explored in the original design. At $2 \mathrm{nC}$ charge [8], and consequently 4 times the temperature rise, electron pulsed heating will lead to surface damage and possibly component failure. Under TESLA FEL parameters, $Q=1 \mathrm{nC}, \sigma_{x}=\sigma_{y}=18 \mu \mathrm{m}, \sigma_{z}=23 \mu \mathrm{m}$ [9], a single bunch pulsed heating of $T_{1}^{\max }=899^{\circ} \mathrm{C}$ and $T_{\text {rad }}=150^{\circ} \mathrm{C}$ on $\mathrm{Ti}$ spoiler can arise. In addition to comparing temperature rise, one needs to keep in mind that electron pulse heating from image current is a surface phenomenon, while radiation heating is mostly a body effect. Therefore the mechanical effects and engineering requirements are different.

The above numerical examples and subsequent observations in the Appendix show that the electron pulse heating due to image currents is an important factor that needs to be considered in future accelerator designs. The example also shows that electron pulsed heating is less important than radiation heating in low peak current machines, which is, perhaps, the reason they are not observed yet.

The calculations in this paper are based on the 1D thermal diffusion equation. For very long pulses and for a very small beam size, i.e., $l_{\text {diff }}^{\text {train }}>\sigma_{x}$, thermal diffusion and energy distribution in transverse $(x)$ direction (Fig. 6) may need to be considered by numerical integration. In such cases, the result in Table I can serve as an upper limit to the maximum temperature rise. Material properties variation with temperature can also be included.

\section{ACKNOWLEDGMENTS}

We would like to acknowledge Josef C. Frisch for suggesting this problem. This work was supported by the Department of Energy under Contract No. DE-AC0376 SF00515.

\section{APPENDIX: OTHER OBSERVATIONS}

The derived expression is also applicable to the case where a beam targets a metal surface at a steep angle, including, but not limited to, OTR screens, profile screens, wire scanners, exit windows, and targets. The surface

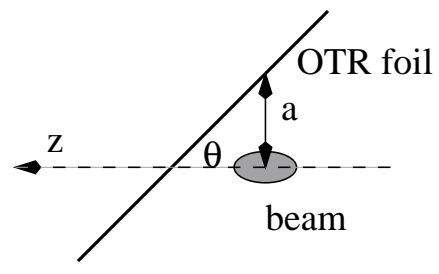

FIG. 10. Beam passes through an OTR screen

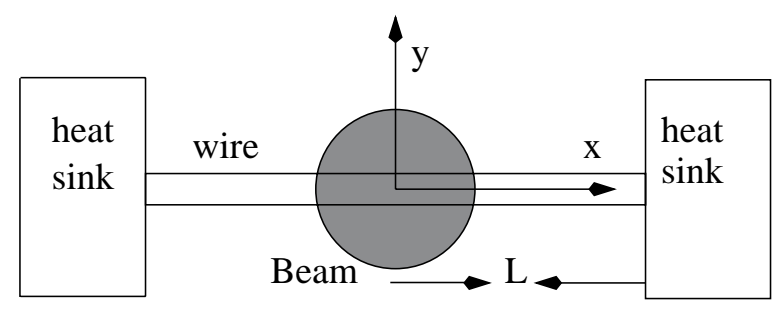

FIG. 11. Beam passes through a wire scanner

magnetic field (Fig. 10) from a point charge is still given by Eq. (3). And the maximum surface magnetic field of a beam, Eq. (23), also applies, as do all the other equations. It is notable that the heating is independent of the angle $\theta$.

Taking a tungsten $\left(\rho C=2.58 \mathrm{~J} / \mathrm{cm}^{3}\right.$ and $d E / d x=$ $22.7 \mathrm{MeV} / \mathrm{cm}$ ) wire scanner at the LCLS as an example (Fig. 11), the pulsed temperature rises due to image currents and radiation are $T_{1}^{\max }=503^{\circ} \mathrm{C}$ and $T_{\text {rad }}=137^{\circ} \mathrm{C}$, respectively. And these are on top of an equilibrium temperature of

$$
T_{\mathrm{rad}}^{\mathrm{eq}}=\frac{(Q / e) d E / d x}{K} \frac{L}{\sqrt{2 \pi} \sigma_{y}} \mathrm{P},
$$

where the wire is assumed to be much smaller than the beam size, and the pulse repetition rate $(\mathrm{P})$ is $120 \mathrm{~Hz}$. The distance $L$ to the heat sink is assumed to be $1 \mathrm{~cm}$. The thermal conductivity of tungsten is $K=146 \mathrm{~W} / \mathrm{m} \cdot \mathrm{K}$ [2]. The total energy deposition from image current is omitted because it is a surface effect. The resulting $T_{\text {rad }}^{\text {eq }}=230^{\circ} \mathrm{C}$ gives a peak temperature rise of $870^{\circ} \mathrm{C}$. Including heat capacity dependency with temperature, $C_{p}=C_{p 0}\left(1+\alpha\left(T-T_{0}\right)\right)$, where $\alpha=2 \times 10^{-4} / \mathrm{K}$ and $T_{0}=300 \mathrm{~K}$, reduces the peak temperature rise to $803^{\circ} \mathrm{C}$. The stress limit of tungsten is $700{ }^{\circ} \mathrm{C}$ [4].

[1] F.-J. Decker et al., in Proceedings of the 18th International Linear Conference (LINAC'96), Geneva, Switzerland, 1996, edited by C. Hill and M. Vretenar (CERN, Geneva, 1996), pp. 137-139.

[2] R. B. Neal et al., The Stanford Two-Mile Accelerator (Benjamin, New York, 1968).

[3] A. P. Prudnikov, Y. A. Brychkov, and O. I. Marichev, Integrals and Serials (Gordon and Breach, New York, 1986).

[4] D. L. Burke et al., Stanford Linear Accelerator Center Technical Report No. SLAC-474, 1996.

[5] D. R. Lide, Handbook of Chemistry and Physics (CRC Press, Boca Raton, FL, 1996), 77th ed. 
[6] R. M. Barnett et al., Phys. Rev. D 54, 1 (1996).

[7] X.E. Lin, in Advanced Accelerator Concepts: Eighth Workshop, edited by Wes Lawson, AIP Conf. Proc. No. 472 (AIP, New York, 1999), pp. 676-685.
[8] M. Cornacchia et al., Stanford Linear Accelerator Center Technical Report No. SLAC-R-521, 1998.

[9] R. Brinkmann et al, DESY Technical Report No. DESY97-048, 1997. 